\title{
Avaliação da qualidade de amostras comerciais de boldo (Peumus boldus Molina), pata-de-vaca (Bauhinia spp.) e ginco (Ginkgo biloba L.)
}

\author{
Melo, J. G. de ${ }^{1}$; Nascimento, V. T. do ${ }^{1}$; Amorim, E. L. C. de ${ }^{2}$; \\ Andrade Lima, C. S. de ${ }^{3}$; Albuquerque, U. P. de ${ }^{1}$ \\ 'Departamento de Biologia, Área de Botânica, Universidade Federal Rural de Pernambuco; \\ ${ }^{2}$ Departamento de Ciências Farmacêuticas; ${ }^{3}$ Departamento de Biofísica e Radiobiologia, \\ Universidade Federal de Pernambuco, Recife, PE.
}

\begin{abstract}
RESUMO: O presente trabalho procurou avaliar a qualidade de produtos comercializados à base de boldo, pata-de-vaca e ginco, através dos parâmetros contidos na Farmacopéia Brasileira e na literatura específica. Foram analisadas oito amostras de boldo, nove de pata-de-vaca e sete de ginco, adquiridas em farmácias na cidade do Recife. A metodologia consistiu em avaliar: os rótulos e bulas dos produtos verificando se estavam de acordo com a RDC no 17 de 24/02/2000 e a portaria 110/97 da ANVISA; realizar análise sensorial; verificar a autenticidade das amostras e sua pureza. Todas as embalagens de boldo, patade-vaca e seis de ginco continham erros ou ausência de informações científicas, além da falta de bula. Na verificação de impurezas, todas as de boldo, cinco de pata-de-vaca, e uma amostra de ginco foram reprovadas. Todos os produtos analisados apresentaram alguma irregularidade segundo os códigos oficiais, sendo necessário uma maior intensificação na vigilância de produtos à base de plantas medicinais no Brasil.
\end{abstract}

Unitermos: controle de qualidade; Peumus boldus Molina; Bauhinia spp.; Ginkgo biloba L.; fitoterápicos; plantas medicinais.

ABSTRACT: Control quality evaluation of commercial samples of boldo (Peumus boldus Molina), pata-de-vaca (Bauhinia spp.) and ginkgo (Ginkgo biloba L.). This study aimed to evaluate the quality of commercial products prepared with boldo (Peumus boldus Molina), pata-de-vaca (Bauhinia spp.) and ginkgo (Ginkgo biloba L.) by using parameters from the Brazilian Pharmacopoeia and specific literature. Eight samples of "boldo", nine of "pata-devaca", and seven of ginkgo were analyzed, all bought from pharmacies in Recife (Pernambuco, Brazil). The methodology consisted in evaluating the products' labels and instructions to verify their accordance to RDC no 17 of 02/24/2000 and ANVISA (National Sanitary Surveillance Agency) decree 110/97, undertaking a sensorial analysis, and verifying the authenticity and purity of the samples. All of the packages of "boldo" and "pata-de-vaca" and six of the packages of ginkgo contained mistakes or lacked scientific information; 
instructions were also missing. After analyzing for impurities, all samples of "boldo", five of "pata-de-vaca", and one of ginkgo were rejected. All of the products analyzed had some sort of irregularity in relation to the official codes, making it necessary to intensify the inspection of medicinal plant products in Brazil.

Key words: quality control; Peumus boldus Molina; Bauhinia spp.; Ginkgo biloba L.; herbal medicinal products; medicinal plants.

\section{INTRODUÇÃO}

Cada país possui um conjunto de regras que regula a produção e a comercialização de drogas vegetais (CALIXTO, 2000). No Brasil, a regulamentação para a comercialização de fitoterápicos é regida por legislação específica, como as portarias da Vigilância Sanitária e por monografias para algumas espécies vegetais, que contêm critérios definidos para identidade, pureza e teor de constituintes químicos.

Embora se tenha parâmetros específicos para a produção e comércio de fitoterápicos, a fraude e a má qualidade têm preocupado profissionais da área de saúde, e a comunidade científica. A ausência de qualidade, a adulteração e a incorreta utilização, interferem na eficácia e até mesmo na segurança do produto, somando-se a isto um serviço de fiscalização não muito eficiente. Conseqüentemente, diversos trabalhos têm sido realizados para alertar sobre a situação atual em que se encontram os fitoterápicos no mundo ( $\mathrm{CHOI}$ et al., 2002; ABOU-ARAB et al., 1999; ELVIN-LEWIS, 2001; ZUCCOLOTTO et al., 1999).

No Brasil, algumas pesquisas têm sido direcionadas para a avaliação de fitoterápicos segundo as normas vigentes. Brandão e colaboradores verificando a qualidade de amostras comerciais de camomila (Matricaria recutita L.), constataram que $96 \%$ possuíam falta de padronização e qualidade (BRANDÃO et al., 1998). Posteriormente, o teste de qualidade com amostras de boldo (Peumus boldus Molina), camomila (Matricaria recutita L.), cidreira (Melissa sp. e Cymbopogon citratus (D.C.) Stapf.), erva doce (Pimpinella anisum L.) e hortelã (Mentha $\mathrm{sp}$.), mostraram grande discordância com as normas vigentes, confirmando que a indústria não segue rigorosamente os princípios de qualidade requeridos para drogas de origem vegetal (BRANDÃO et al., 2002).

As espécies Peumus boldus Molina (boldo), Ginkgo biloba L. (ginco), e as plantas do gênero Bauhinia (pata-de-vaca) são bastante difundidas na cultura medicinal brasileira, e freqüentemente encontradas na composição de fitoterápicos industrializados, além de serem comercializados em feiras livres em todo território nacional.

O gênero Bauhinia possui aproximadamente 300 espécies (SILVA; FILHO, 2002), pertence a família Leguminosae e é conhecida popularmente como pata-de-vaca ou mororó (MATOS, 1998). Para algumas espécies de Bauhinia são atribuídas propriedades antifúngicas, antibacterianas, antiinflamatórias, e em especial antidiabética (SILVA; FILHO, 2002).

A espécie Ginkgo biloba L. é empregada na medicina tradicional chinesa há séculos, sendo referida como um fóssil vivo. É a única integrante da família Ginkgoaceae. No Brasil o seu uso é recente, sendo usada principalmente para promover maior circulação sangüínea no cérebro, indicada nas disfunções cerebrais, vertigens e para melhorar a concentração e a memória (LORENZI; MATOS, 2002).

Peumus boldus Molina pertence a família Monimiaceae e tem como nome popular boldo ou boldo-do-chile. É usada para o tratamento de distúrbios hepáticos e colelitíase, tendo ainda propriedades diuréticas e antiinflamatórias (MATOS, 1998).

Rev. Bras. Farmacogn., V. 14, n. 2, jul.-dez. 2004. 
O presente trabalho teve por objetivo avaliar a qualidade de produtos à base de boldo, ginco e pata-de-vaca comercializados em farmácias da cidade do Recife, analisando as informações fornecidas e se as amostras encontravam-se de acordo com as exigências da legislação específica.

\section{MATERIAL E MÉTODOS}

Através de um levantamento realizado em 54 farmácias na cidade do Recife, entre março e agosto de 2002, foram selecionados produtos a base de três plantas medicinais de grande ocorrência para realizar o controle de qualidade. Vinte e quatro marcas comerciais diferentes foram adquiridas, sendo oito de boldo, nove de pata-de-vaca e sete de ginco.

\section{Análise de rótulos e bulas}

Foram analisadas a correta nomenclatura botânica oficial (gênero, espécie, autor do binômio e família), se a composição vegetal do produto condizia com o rótulo ou bula, a parte da planta utilizada e o estado em que se encontrava a amostra, o peso real do produto, e outros requisitos contidos na RDC o17 (BRASIL, 2000). Todos os rótulos e bulas foram analisados quanto a presença de frases e dizeres obrigatórios por lei (BRASIL, 1997).

\section{Análise sensorial}

Para os produtos a base de boldo foram observadas as características sensoriais (FARMACOPÉIA BRASILEIRA, 1959). Para os produtos a base de pata-de-vaca e ginco, analisouse apenas o seu aspecto de conservação, uma vez que não se encontrou referências para estas drogas vegetais.

\section{Autenticidade da amostra}

A autenticidade das amostras foi avaliada através de parâmetros contidos na Farmacopéia Brasileira (1959 e 2000) e na literatura específica (TESKE; TRENTINI, 1997; OLIVEIRA et al., 2001; SILVA; FILHO, 2002; MATOS, 1995).

Para análise dos caracteres microscópicos, folhas ou fragmentos destas foram hidratadas e em seguida secionadas transversalmente no terço médio e nas bordas com o auxílio de medula de embaúba como suporte. Os cortes foram clarificados em solução de água e hipoclorito de sódio (20:1). O material foi corado em safrablau (azul de alcian 1\% + safranina 1\%, 9:1) ou Etzold (ETZOLD, 1983). No caso de drogas pulverizadas a visualização foi feita diretamente ao microscópio e comparadas com amostra autêntica.

Os testes fitoquímicos realizados visaram evidenciar as principais classes de substâncias químicas presentes nas espécies, por reações qualitativas, a partir de extratos das plantas com reagentes específicos para cada classe (MATOS, 1995). Os extratos foram preparados por maceração a partir de 5 gramas de cada amostra em etanol por quarenta e oito horas. Em seguida, o extrato foi filtrado e foram realizados os testes fitoquímicos. As classes de substâncias testadas foram: fenóis, taninos hidrolisáveis e condensados, flavonóides, antocianinas e antocianidinas, catequinas e leucoantocianidinas, e alcalóides.

Também realizou-se cromatografia em camada delgada (CCD) para a confirmação da presença de alcalóides para os produtos a base de pata-de-vaca e boldo, usando a ioimbina como referência, com o sistema de eluição acetato de etila, ácido fórmico, ácido acético e água 
destilada (100:11:11:27 v/v), e usando como revelador Dragendorff (WAGNER; BLANDT, 1996). Para os produtos a base de Ginco, investigou-se a presença de flavonóides, sendo usado a quercetina como padrão, com o mesmo sistema de eluição descrito acima (WAGNER; BLANDT, 1996).

\section{Verificação de pureza}

A verificação da pureza baseou-se na pesquisa de elementos estranhos, determinação de cinzas totais e do teor de umidade, comparando-se os resultados encontrados com o indicado pelas monografias farmacopéicas (FARMACOPÉIA BRASILEIRA, 2000; FARMACOPÉIA AMERICANA, 2001).

\section{RESULTADOS}

\section{Análise de rótulos e bulas}

Dentre os vinte e quatro produtos fitoterápicos analisados, vinte e três apresentaram peso conforme o especificado na embalagem (exceto uma amostra de boldo), treze não informavam o estado em que se encontrava o material, sendo dentre estes, cinco à base de boldo, cinco à base de pata-de-vaca e três a base de ginco. Um produto à base de boldo, três constituídos por pata-de-vaca e dois por ginco não mencionavam a parte da planta utilizada. Apenas um produto composto por ginco apresentou as informações na bula, sendo que todos os demais produtos à base de boldo e pata-de-vaca possuíam somente rótulos ou etiquetas com informações incompletas, infringindo a portaria 110/97 da ANVISA que determina a obrigatoriedade da inclusão da bula nas embalagens dos produtos.

As grafias do gênero e do epiteto específico de todas as amostras dos medicamentos a base de boldo, três de pata-de-vaca, e três de ginco, encontravam-se erradas, infringindo as regras botânicas. A correta grafia da espécie, a citação da família e do autor do binômio, embora a legislação específica exija a menção na bula (BRASIL, 2000), só foram encontradas de maneira irrepreensível em um dos produtos (ginco), tendo os demais vinte e três produtos violado a resolução. Os erros mais comuns no tocante ao nome científico dos produtos à base de boldo foram "Pneumus boldo Molina" ou "Peumos boldos M." Para os produtos à base de pata-de-vaca a citação mais comum foi "Bautirea festicapa", sendo o nome correto para a espécie Bauhinia forficata Link. Para os produtos constituídos por ginco a inscrição mais comum para citar a espécie foi "Ginko biloba".

A designação "MEDICAMENTO FITOTERÁPICO" ou "MEDICAMENTO FITOTERÁPICO TRADICIONAL" que deveria constar nos rótulos dos produtos, foi negligenciada pela grande maioria (com exceção de uma amostra a base de ginco). Com relação a presença das frases obrigatórias: "Informe ao seu médico a ocorrência de gravidez na vigência do tratamento ou após o seu término"; "Informe ao seu médico o aparecimento de reações desagradáveis"; "Não tome remédio sem o conhecimento do seu médico, pode ser perigoso para sua saúde", observou-se que apenas 12,5\% (exatamente três medicamentos, à base de Ginkgo biloba L.) dos fitoterápicos analisados apresentavam de 2-5 frases obrigatórias, sendo a grande maioria dos produtos, todos à base de boldo, pata-de-vaca e quatro à base de ginco, infratores da portaria $110 / 97$ da ANVISA. Cerca de $25 \%$ dos produtos continham dizeres como: produto natural, cem por cento natural ou produto naturalmente saudável, caracterizando frases indutoras de consumo.

De um total de vinte e quatro amostras analisadas, apenas um produto à base de ginco apresentou-se em concordância com as exigências específicas. Inversamente, seis produtos à

Rev. Bras. Farmacogn., V. 14, n. 2, jul.-dez. 2004. 
base de ginco, todos à base de boldo e pata-de-vaca, totalizando 95,8\%, apresentavam algum tipo de irregularidade (Tabela 1).

Tabela 1. Principais causas de rejeição dos fitoterápicos à base de boldo, pata-de-vaca e ginco comercializados em farmácias de Recife-PE.

\begin{tabular}{l|c|}
\multicolumn{1}{|c|}{ CAUSA DA REJEIÇÃO } & PERCENTUAL (\%) \\
\hline Ausência de bulas & 95,83 \\
$\begin{array}{l}\text { Falta ou insuficiência de informações } \\
\text { obrigatórias }\end{array}$ & 95,83 \\
Ausência de um dos constituintes químicos & 20,83 \\
Excesso de constituintes indesejáveis & 54,16 \\
\hline
\end{tabular}

\section{Análise sensorial}

Todas amostras de boldo observadas se apresentaram adequadas quanto a cor e odor que caracterizam a espécie, não sendo encontrada, discordância quanto às características descritas na norma vigente para esta análise. Para os produtos à base de pata-de-vaca, duas amostras apresentaram cor e estado de conservação fora dos padrões (em decomposição). Para as amostras de ginco não se encontrou alterações odoríferas e cromáticas.

\section{Autenticidade das amostras}

As características macro e microscópicas remetiam a espécie mencionada na embalagem, ou seja, todas as amostras de boldo correspondiam à espécie Peumus boldus Molina, as de ginco eram constituídas por Ginkgo biloba L., e as de pata-de-vaca correspodiam ao gênero Bauhinia.

$\mathrm{Na}$ análise microscópica dos produtos a base de boldo, foram observadas em todas as amostras estruturas celulares características da espécie (FARMACOPÉIA BRASILEIRA, 1959). Os pêlos epidérmicos na face adaxial e as glândulas oleíferas de grande volume e forma de globo só foram vistas em quatro amostras, pois duas se encontravam bastante pulverizadas dificultando a visualização.

Nos produtos a base de pata-de-vaca a análise macroscópica foi realizada em quatro amostras, pois as restantes encontravam-se pulverizadas. Todas as quatro amostras referidas acima foram confirmadas como pertencentes ao gênero Bauhinia. Nas amostras pulverizadas de pata-de-vaca foi possível observar algumas estruturas como pêlos tectores, parênquima, tecidos da epiderme, e cristais prismáticos. Todas as amostras apresentaram características que remetem ao gênero supracitado (OLIVEIRA et al., 2001).

Das sete amostras de ginco, quatro apresentaram-se pulverizadas e três eram constituídas de folhas inteiras. Todas as amostras apresentaram estruturas características da espécie citada.

Os testes fitoquímicos comprovaram a existência de fenóis, taninos condensados, flavonóides, catequinas (cinco amostras) e alcalóides em todas as amostras de boldo; fenóis, taninos condensados, flavonóides, catequinas e alcalóides (sete amostras) para os produtos a base de pata-de-vaca; e fenóis, flavonóides e catequinas para os produtos a base de Ginkgo biloba L. 


\section{Verificação da pureza}

Todas as amostras analisadas no requisito elementos estranhos foram reprovadas, pois embora não se tenha parâmetros definidos para estas espécies, os índices superaram o permitido (Tabelas 2 e 3) pela Farmacopéia Brasileira (2000) que é de no máximo 2\%, para a maioria das drogas vegetais.

Tabela 2. Teorés de elementos estranhos, umidade e cinzas totais em produtos à base de boldo (B), pata-de-vaca $(P)$ e ginco $(G)$, comercializados em farmácias da cidade do Recife-PE.

\begin{tabular}{|c|c|c|c|c|}
\hline Amostras & Elementos estranhos (\%) & Teor de umidade (\%) & Cinzas Totais (\%) & Situação \\
\hline B1 & 6,02 & 7,25 & 13,29 & reprovada \\
\hline B2 & 3,94 & 8,10 & 11,29 & reprovada \\
\hline B3 & 6,91 & 8,32 & 11,94 & reprovada \\
\hline B4 & 4,67 & 5,91 & 15,27 & reprovada \\
\hline B5 & 7,83 & 6,97 & 11,48 & reprovada \\
\hline B6 & 2,93 & 7,63 & 11,89 & reprovada \\
\hline B7 & 6,58 & 8,68 & 11,86 & reprovada \\
\hline B8 & 7,98 & 10,07 & 12,82 & reprovada \\
\hline $\mathrm{P} 1$ & 54,56 & 6,49 & 5,09 & reprovada \\
\hline $\mathrm{P} 2$ & 37,43 & 8,42 & 2,82 & reprovada \\
\hline P3 & $\#$ & 6,45 & 6,27 & aprovada \\
\hline $\mathrm{P} 4$ & 6,00 & 8,30 & 2,73 & reprovada \\
\hline P5 & 55,5 & 8,42 & 5,50 & reprovada \\
\hline P6 & 22,75 & 7,74 & 5,01 & reprovada \\
\hline $\mathrm{P} 7$ & * & 8,05 & 4,67 & aprovada \\
\hline P8 & $\#$ & 7,97 & 8,16 & aprovada \\
\hline P9 & $\#$ & 5,02 & 5,63 & aprovada \\
\hline G1 & * & 12,70 & 7,86 & reprovada \\
\hline G2 & $\#$ & 6,52 & 7,33 & aprovada \\
\hline G3 & $\#$ & 10,39 & 8,05 & aprovada \\
\hline G4 & $\#$ & 8,61 & 6,65 & aprovada \\
\hline G5 & \# & 6,41 & 9,52 & aprovada \\
\hline G6 & * & 6,61 & 8,43 & aprovada \\
\hline G7 & * & 5,93 & 7,65 & aprovada \\
\hline
\end{tabular}

* Produto sem elementos estranhos visíveis a olho nu.

\# material pulverizado

Rev. Bras. Farmacogn., V. 14, n. 2, jul.-dez. 2004. 
Tabela 3. Amostras rejeitadas de fitoterápicos à base de boldo, pata-de-vaca e ginco, comercializados em farmácias de Recife-PE.

\begin{tabular}{|c|c|c|c|}
\hline PLANTA & $\begin{array}{l}\text { № DE AMOSTRAS } \\
\text { ANALISADAS }\end{array}$ & $\begin{array}{l}\text { PERCENTUAL DE } \\
\text { REJEIÇÃO (\%) }\end{array}$ & CAUSAS DA REJEIÇÃO \\
\hline Boldo & 8 & 100 & $\begin{array}{c}\text { Ausência de bulas, e } \\
\text { insuficiência nas } \\
\text { informações/ Elementos } \\
\text { indesejáveis. }\end{array}$ \\
\hline Ginco & 7 & 85,71 & $\begin{array}{l}\text { Ausência de bulas, e } \\
\text { insuficiência nas } \\
\text { informações. }\end{array}$ \\
\hline Pata-de-vaca & 9 & 55,55 & Elementos indesejáveis. \\
\hline
\end{tabular}

Foram encontrados em todos os produtos à base de boldo impurezas constituídas por pequenos galhos, frutos e pecíolos, pedúnculos, e estruturas que se assemelhavam a palha nas sete amostras trituradas. Também foram encontrados materiais de origem mineral, como pequenas pedras e areia. A verificação da pureza ficou prejudicada nas cinco amostras trituradas, pois alguns constituintes não-desejáveis quando visualizados a estereomicroscópio possuíam diâmetro muito pequeno, sendo difícil sua separação.

Nos produtos à base de pata-de-vaca, ainda que não seja preconizada a parte vegetal nos códigos oficiais, considera-se a folha como parte a ser utilizada para fins terapêuticos, uma vez que é o órgão vegetal utilizado na medicina popular. Os elementos estranhos eram constituídos por gramíneas, estruturas semelhantes a palha, pequenas pedras e principalmente estruturas lenhosas. Em quatro amostras não foi possível esta análise devido ao estado pulverizado do produto.

Dentre as sete amostras de ginco, três estavam sem elementos estranhos visíveis a olho nu, e quatro encontravam-se pulverizadas impossibilitando a análise.

A umidade de um produto à base de ginco ultrapassou o estabelecido pela Farmacopéia Americana (2001), que preconiza em $11 \%$ o percentual máximo de água.

Todas as amostras de boldo ultrapassaram o limite estabelecido pela Farmacopéia Brasileira (2000) para o teor de cinzas totais (10\%). Já os produtos à base de ginco não ultrapassaram o limite estabelecido (11\%) pela Farmacopéia Americana (2001). Para os produtos à base de patade-vaca não foi possível uma análise exata por tratar-se de um gênero, ocorrendo uma variação no caso de diferentes espécies, além da falta de referência para esta análise na literatura.

\section{DISCUSSÃO E CONCLUSÃO}

De acordo com os resultados obtidos na análise de rótulos e bulas, os produtos comercializados no Recife a base de boldo, pata-de-vaca e ginco carecem de uma padronização de suas informações, e de uma correta conduta perante a legislação específica, uma vez que $95,83 \%$ dos produtos analisados foram reprovados.

A ausência de bulas e as informações científicas incompletas e imprecisas em vinte e três produtos testificam o descaso que é dado as informações técnicas indispensáveis ao consumidor. Bello et al. (2002) em análise de bulas de 65 medicamentos fitoterápicos em Porto Alegre, verificaram que $51 \%$ não possuíam bula e que as informações eram deficientes, não seguindo as normas legais. Vem a contribuir para tal quadro, nos principais centros urbanos do Brasil, a 
comercialização de chás medicinais com fins alimentícios (BRASIL, 1998), sendo neste caso, os produtos relacionados como simples alimento, não havendo obrigatoriedade da presença da bula. Porém, o que se observa é que algumas espécies vegetais são aprovadas para serem comercializadas como alimento tendo propriedades terapêuticas comprovadas como, por exemplo, o boldo (Peumus boldus Molina). No entanto, existe uma contradição em classificar uma planta medicinal como um simples produto alimentício, pois para a maioria das espécies vegetais comercializadas como tal, há comprovação da atividade terapêutica e para algumas há contraindicações. É o caso do boldo, que possui efeitos tóxicos pela presença do ascaridol, que pode levar a complicações para a saúde do consumidor, como irritação renal, podendo ser prejudicial durante a gestação (NEWAL et al. apud MARQUES; PETROVICK, 2001). Além disso, o extrato das folhas de boldo, bem como a boldina, mostraram ação abortiva e teratogênica, e alterações dos níveis sangüíneos de colesterol, glicose e uréia em ratos (ALMEIDA et al., 2000).

Apesar da exigência da RDC no17 de 24/02/2000, para que a bula do produto fitoterápico contenha nomenclatura botânica oficial, a composição explícita na embalagem e a correspondência de marcadores e/ou princípios ativos, e da portaria 110/97 da ANVISA que exige a presença de frases obrigatórias, o que se observa é a infração destas normas pela indústria. Embora a literatura (TESKE; TRENTINI, 1997; SILVA; FILHO, 2002) divulgue a correspondência de marcadores e princípios químicos ativos das espécies vegetais analisadas neste trabalho, e a legislação ordene a descrição na bula de tais elementos, somente um medicamento a base de ginco apresentou tal especificação, tendo os demais transgredido a RDC nำ17 no item 7.2.3.

Não obstante as informações científicas divulgadas referente às propriedades farmacológicas dos produtos analisados, como o poder diurético e hipoglicemiante de Bauhinia forficata Link, e as reações adversas com a superdosagem do Peumus boldus Molina, que pode provocar alucinações cromáticas e auditivas, tonturas, vômitos, diarréias e até convulsões (TESKE; TRENTINI, 1997), reforça-se que as informações específicas como ação do fitoterápico, contraindicações, precauções, reações adversas, interações medicamentosas, modos de administração e cuidados no armazenamento, devem estar presentes na bula destes produtos. Da maioria das amostras aqui apresentadas, 95,83\%, não continham essas informações completas, podendo levar ao uso indevido, alterações das funções orgânicas no estado natural do indivíduo, e trazer prejuízos para a saúde do mesmo.

Cinco produtos de pata-de-vaca citavam apenas o gênero sem uma definição da espécie (Bauhinia sp.), apresentando um erro grave, uma vez que para este gênero existem aproximadamente 300 espécies distribuídas pelas regiões tropicais, subtropicais e temperadas do mundo, especialmente na América do Sul (SILVA; FILHO, 2002), sendo que nem todas as espécies deste gênero possuem poder terapêutico comprovado e/ou em estudo, como conhecido para Bauhinia forficata Link.

A reação negativa no teste de investigação fitoquímica para catequinas em três amostras de boldo, e para alcalóides em duas amostras de pata-de-vaca, sugere ausência ou baixa concentração destas classes de constituintes, possivelmente devido a interferência ambiental como temperatura, umidade, tipo de solo, estágio de desenvolvimento entre outros (PIRES; CHICOUREL, 1997), tendo como possível conseqüência um prejuízo da atividade terapêutica (MOREIRA, 2003).

Os índices superiores ao permitido de matéria estranha para os produtos, se devem possivelmente ao manejo, limpeza e separação inadequadas, sendo um problema freqüente nos produtos a base de plantas medicinais comercializados no Brasil (ZUCCOLOTTO et al., 1999; BARBOSA et al., 2001; AMARAL et al., 2003; BRANDÃO et al., 2002). A presença de um alto teor de elementos estranhos nos produtos analisados compromete a qualidade da droga, interferindo na sua eficácia, pondo em risco a saúde do consumidor.

Rev. Bras. Farmacogn., V. 14, n. 2, jul.-dez. 2004. 
Os teores de umidade não foram considerados altos, pois estavam abaixo do limite máximo permitido (8-14\%) na Farmacopéia Brasileira (2000). Os índices superiores para as amostras de boldo, no teor de cinzas totais, indica uma possível contaminação dos produtos por impurezas de origem inorgânica.

Conclui-se que os produtos a base de boldo, pata-de-vaca e ginco comercializados na cidade do Recife carecem de bulas uniformizadas com informações técnicas completas e indispensáveis para o consumidor; os fitoterápicos a base de boldo e pata-de-vaca podem oferecer riscos ao usuário, uma vez que não estão adequados as exigências sanitárias; faz-se necessário a inclusão da pata-de-vaca (Bauhinia forficata Link) nas próximas edições da Farmacopéia Brasileira, para fornecer subsídios para o controle de qualidade, visando assegurar a segurança dos produtos; é de grande importância a adequação da legislação no combate a dubiedade de normas específicas para o registro, bem com uma forte intensificação da vigilância por parte dos órgãos competentes, para a obtenção de produtos seguros e eficazes.

\section{AGRADECIMENTOS}

À Fundação de Amparo a Ciência e Tecnologia do Estado de Pernambuco (FACEPE) pelo apoio financeiro, e ao PIBIC/CNPq/UFRPE pela concessão de bolsas de iniciação científica.

\section{REFERÊNCIAS}

ABOU-ARAB, A.A.K.; KAWTHER, M.S.; TANTAWY, M.E.EI.; BADEAA, R.I.; KHAYRIA N. Quantity estimation of some contaminants in commonly used medicinal plants in the Egyptian market. Food Chemistry, v.67, n.1, p.357-363, 1999.

ALMEIDA, E.R.; MELO, A.M.; XAVIER, H. Toxicological evaluation of the hydro-alcohol extract of the dry leaves of Peumus boldus and boldine in rats. Phytoterapy Research, v.14, n.2, p.99-102, 2000.

AMARAL, F.M.M.; COUTINHO, D.F.; RIBEIRO, M.N.S.; OLIVEIRA, M.A. Avaliação da qualidade de drogas vegetais comercializadas em São Luís/ Maranhão. Revista Brasileira de Farmacognosia, v.13, n.1, p.2730, 2003.

BARBOSA, M.C.S.; BELLETTI, K. M. da S.; CORRÊA, T. F.; SANTOS, C.A. de M. Avaliação da qualidade de folhas de boldo-do-chile (Peumus boldus Molina) comercializadas em Curitiba, PR. Revista Brasileira de Farmacognosia, v.11, n.1, p.1-4, 2001.

BELLO, C.M.; MONTANHA, J.A.; SCHENKEL, E.P. Análise das bulas de medicamentos fitoterápicos comercializados em Porto Alegre, RS, Brasil. Revista Brasileira de Farmacognosia, v.12, n.2, p.75-83, 2002.

BRANDÃO, M.G.L.; FREIRE, N.; SOARES, C.D.V. Vigilância de fitoterápicos em Minas Gerais: Verificação da qualidade de diferentes amostras comerciais de camomila. Caderno de Saúde Pública, v.14, n.3, p.613-616, 1998.

BRANDÃO, M.G.L.; ALVES, R.M.S.; MOREIRA, R.A.; OLIVEIRA, P.; VIEIRA, M.T.; MOREIRA-CAMPOS, L.M. Qualidade de amostras comerciais de chás de plantas medicinais. Revista Brasileira de Plantas Medicinais, v.5, n.1, p.56-59, 2002.

BRASIL. Ministério da saúde. Agência Nacional de Vigilância Sanitária. Portaria no 110 de 10 de março de 1997. Trata das informações obrigatórias que deve conter as bulas de medicamentos. Disponível em: <http:// www.anvisa.gov./br/legis/portarias/110_97.htm. Acesso em: 02 nov. 2002.

BRASIL. Ministério da saúde. Agência Nacional de Vigilância Sanitária. Portaria no 519 de 26.06.1998. Regulamento técnico para fixação de identidade de chás - Plantas destinadas 1 a preparação de infusões ou decoctos. Diário Oficial da União, 29.06.1998.

BRASIL. Resolução da Diretoria Colegiada (RDC) ㄲo 17, de 24 de fevereiro de 2000. Dispõe sobre o registro de medicamentos fitoterápicos. Diário Oficial da União, seção 1, p.25, 2000. 
CALIXTO, J.B. Efficacy, safety, quality control, marketing and regulatory guidelines for herbal medicines (Phytotherapeutic agents). Brazilian Journal of Medical and Biological Research, v.33, n.1, p.179-189, 2000.

CHOI, D.W.; KIM, J.H.; CHO, SO Y.; KIM, D.H.; CHANG, S.Y. Regulation and quality control of herbal drugs in Korea. Toxicology, v.181-182, n.1, p. 581-586, 2002.

ELVIN-LEWIS, M. Should we be concerned about herbal remedies. Journal of Ethnopharmacology, v.75, n.1, p.141-164, 2001.

ETZOLD, H. Eine Kontrastreiche simultane Mehrfachfärbung für pflanzenanatomische Präparate.. Mikroskosmos, v.72, p.213-218, 1983.

FARIAS, M.R. Avaliação da qualidade de matérias-primas vegetais. In: SIMÕES, C.M.O. et al. (org.) Farmacognosia: da planta ao medicamento. 3.ed., Florianópolis: Ed. UFSC, p. 199-222, 2001.

FARMACOPÉIA AMERICANA. USP. 25. Toronto: The United States Pharmacopeial Convention, 2001.

FARMACOPÉIA BRASILEIRA. 4.ed. Parte II. São Paulo: Atheneu, 2000.

FARMACOPÉIA BRASILEIRA. 2.ed. São Paulo: Siqueira, 1959.

LORENZI, H.; MATOS, F. J. A. Plantas Medicinais no Brasil: Nativas e Exóticas. Nova Odessa: Instituto Plantarum, 2002.

MARQUES, L. C.; PETROVICK, P. R. Normatização da produção e comercialização de fitoterápicos no Brasil. In: SIMÕES, C.M.O. et al. (org.) Farmacognosia: da planta ao medicamento. 3.ed., Florianópolis: Ed. UFSC, p.261-299, 2001.

MATOS, F.J.A. Introdução a fitoquímica experimental. Fortaleza: EUFC, 1995.

MATOS, F.J.A. Farmácias Vivas. Fortaleza: EUFC, 1998.

MOREIRA, E. A. A Farmacognosia e a farmacopéia brasileira. Jornal Brasileiro de Fitomedicina, v.1, n.1, p.04-05, 2003.

OLIVEIRA, F.; KATO, E.T.M.; RODRIGUES, R.F.O.; BASSO, S.L. Mitos e verdades sobre pata-de-vaca Bauhinia forficata Link - Uma revisão. Revista Lecta, v.19, n.1, p.07-20, 2001.

PIRES, C.A.; CHICOUREL, E. L. Metodologias para controle de qualidade de plantas medicinais do horto da FFB/UFJF. Principia: Caminhos da Iniciação Científica, v.2, n.1, p.217-225, 1997.

SILVA, M.L.; FILHO, V.C. Plantas do gênero Bauhinia: composição química e potencial farmacológico. Química Nova, v.25, n.3, p.449-454, 2002.

TESKE, M.; TRENTINI, A.M.M. Herbarium: Compêndio de fitoterapia. Curitiba: Herbarium, 1997.

ZUCCOLOTTO, T.; APEL, M.; RATES, S.M.K. Avaliação da qualidade de produtos fitoterápicos comercializados em Porto Alegre- RS. Revista do Instituto Adolfo Lutz, v.58, n.2, p.25-31, 1999.

WAGNER, H.; BLANDT, S. Plant drug analysis. 2.ed., New York: Springer, 1996.

*Autor para correspondência

Prof. Dr. Ulysses Paulino de Albuquerque

Laboratório de Etnobotânica Aplicada

Departamento de Biologia

Universidade Federal Rural de Pernambuco

52171-030 - Recife - PE

E-mail: upa@ufrpe.br

Rev. Bras. Farmacogn., V. 14, n. 2, jul.-dez. 2004. 